\title{
A NOTE ON MONOTONE DEFORMATION-FREE MAPPINGS
}

\section{L. CURTIS}

If $M$ is a closed subset of a space $S$ and $h: M x I \rightarrow S$ is a deformation, then $h$ is called a deformation-free mapping of $M$ into $S$ if, for each $0 \leqq t \leqq 1, h(M x t) \cap M=0$. In the case $M$ is a locally simply connected continuum separating the $n$-sphere $S^{n}, A$ is a component of $S^{n}-M$, and $M$ is deformation free into $\bar{A}$ with a monotone open deformation-free map, then $A$ is uniformly locally simply connected $[1$, Theorem 3.1]. The purpose of this note is to show that the condition that $h$ be open can be omitted.

The first lemma is a standard result in the theory of covering spaces. The second lemma is a simple consequence of the first (also noted by G. D. Mostow [2] in the case $\left.\pi_{1}(X)=0\right)$.

Lemma 1. Let $X$ and $Y$ be arcwise connected and locally arcwise connected topological spaces. Let $\tilde{Y}$ be a covering of $Y$ with projection $p: \tilde{Y} \rightarrow Y$ and let $f: X \rightarrow Y$ be onto. If $p_{*}\left(\pi_{1}(Y)\right) \supset f_{*}\left(\pi_{1}(X)\right)$, then there exists a map $g: X \rightarrow \tilde{Y}$ such that $p g=f$. ( $p_{*}$ and $f_{*}$ denote the maps induced on the fundamental groups by $p$ and $f$ respectively.)

LemmA 2. If $X$ and $Y$ are as in Lemma 1 and

(1) $Y$ is locally simply connected,

(2) $f: X \rightarrow Y$ is monotone and onto, then $f_{*}: \pi_{1}(X) \rightarrow \pi_{1}(Y)$ is onto.

Proof. Take a covering $\tilde{Y}$ of $Y$ such that $p_{*}\left(\pi_{1}(\tilde{Y})\right)=f_{*}\left(\pi_{1}(X)\right)$ and factor $f$ as in Lemma 1. Define a map $\beta: Y \rightarrow \tilde{Y}$ as follows: If $y \in Y$, then $f^{-1}(y)$ is a connected set ( $f$ is monotone) so that $g\left(f^{-1}(y)\right)$ is also connected. Since $g\left(f^{-1}(y)\right)$ is contained in the discrete set $p^{-1}(y)$, it is a single point, and the definition $\beta(y)=g\left(f^{-1}(y)\right)$ gives a singlevalued function. It is almost immediate that $\beta$ is one-to-one, continuous and open. Since $\tilde{Y}$ is a covering of $Y$, this implies that $\tilde{Y}=Y$, $p$ is the identity, and $\pi_{1}(Y)=\pi_{1}(\tilde{Y})=f_{*} \pi_{1}(X)$, so that $f_{*}$ is onto.

Theorem. If $M$ is a locally simply-connected continuum which separates $S^{n}$ and which is deformation free into $\bar{A}$ ( $A$ a component of $\left.S^{n}-M\right)$ with a monotone deformation-free mapping, then $A$ is uniformly locally simply connected.

Proof. Let $h: M x I \rightarrow \bar{A}$ be a monotone deformation-free mapping.

Presented to the Society, April 25, 1953; received by the editors July 13, 1953 and, in revised form, September 27, 1953. 
Since $\bar{A}$ is compact, showing $A$ to be uniformly simply connected is equivalent to showing $A$ to be locally simply connected relative to $\bar{A}[3]$. Since $A$ is locally simply connected, one must simply show that given $\epsilon>0$ and $x \in M$, there exists a $\delta>0$ such that any continuous 1 -sphere in $S(x, \delta) \cap A$ is nullhomotopic in $S(x, \epsilon) \cap A$. $(S(x, \alpha)$ means the open ball of radius $\alpha$ with center $(x, 0)$.)

Let $R(x, \zeta)$ denote the set of points in $M x I-M x 0$ which are a distance less than $\zeta$ from $x$, using a metric in $S x I$. Since $h$ is a deformation-free map and $M$ is locally simply-connected, it is easy to show that one can choose $\delta^{\prime}>0$ so that any continuous 1 -sphere in $R\left(x, \delta^{\prime}\right)$ is homotopic to a point in $h^{-1}(S(x, \epsilon) \cap A)$. Choose $\delta>0$ so that $R\left(x, \delta^{\prime}\right)$ contains $h^{-1}(S(x, \delta) \cap A)$.

Let $f\left(S^{1}\right)$ be a continuous 1 -sphere in $S(x, \delta) \cap A$. Let $T$ be a connected open set in $S(x, \delta) \cap A$ such that $f\left(S^{1}\right) \subset T$. Since $h$ is monotone $h^{-1}(T)$ is connected, and $h^{-1}(T) \subset R\left(x, \delta^{\prime}\right)$ by the choice of $\delta$. Letting $h^{-1}(T)=X, T=Y$ and $h \mid h^{-1}(T)=f$, the conditions of Lemma 2 are satisfied. $\left(h^{-1}(T)\right.$ is locally arcwise connected because $M$ is, and $M$ is by Theorem 1.1 in [1]). Hence there is a map $f^{\prime}$ which is homotopic (in $T$ ) with $f$ and a map $g: S^{1} \rightarrow h^{-1}(T)$ such that $f^{\prime}=h g$. Now $g\left(S^{1}\right)$ is nullhomotopic in $h^{-1}(S(x, \epsilon) \cap A)$ so that $f\left(S^{1}\right)$ is nullhomotopic in $S(x, \epsilon) \cap A$ and the theorem is proved.

\section{BIBLIOGRAPHY}

1. M. L. Curtis, Deformation-free continua, Ann. of Math. vol. 57 (1953) pp. 231247.

2. G. D. Mostow, A theorem on locally Euclidean groups, Proc. Amer. Math. Soc. vol. 2 (1951) pp. 285-289.

3. S. Eilenberg and R. L. Wilder, Uniform local connectedness and contractibility, Amer. J. Math. vol. 44 (1942) pp. 408-426.

NORTHWESTERN UNIVERSITY 